\title{
基于应力场的锂离子电池正极多尺度失效研究
}

\author{
陈 莹 ${ }^{1}$, 栾伟玲 ${ }^{1}$, 陈浩峰 ${ }^{1,2}$, 朱轩辰 ${ }^{2}$
}

(1. 华东理工大学 机械与动力工程学院, 石化行业动力电池系统与安全重点实验室, 教育部承压系统与安全重点 实验室,上海 200237; 2. 思克莱德大学 机械与航空工程系, 格拉斯哥 G11XJ 英国)

摘 要: 锂离子电池已广泛应用于动力和储能领域, 电池寿命是影响其进一步发展的关键因素。循环充放电过程中 的电化学-力学多场耦合作用会导致正极材料发生机械损伤累积, 降低电极材料的结构稳定性并形成多尺度损伤, 从而缩短电池循环充放电寿命。本文通过总结团队在三元正极材料多尺度失效行为方面的研究成果, 系统介绍了不 同尺度下实验与模拟相结合的电极材料损伤分析方法, 旨在为不同尺度下选取损伤分析方法提供参考。基于电化学 循环实验表征、扩展有限元分析法(XFEM)、线性匹配法(LMM)等研究手段，深入分析了电极材料在多尺度下的力 学损伤机理。研究工作为电极材料的多尺度失效行为分析及结构改性提供了重要指导。

关 键 词: 锂离子电池; 正极; 应力场; 多尺度失效; 寿命衰减

中图分类号: TM911 文献标志码: A

\section{Multi-scale Failure Behavior of Cathode in Lithium-ion Batteries Based on Stress Field}

\author{
CHEN Ying ${ }^{1}$, LUAN Weiling ${ }^{1}$, CHEN Haofeng $^{1,2}$, ZHU Xuanchen $^{2}$
}

(1. Key Laboratory of Power Battery Systems and Safety (CPCIF), Key Laboratory of Pressure Systems and Safety (MOE), School of Mechanical and Power Engineering, East China University of Science and Technology, Shanghai 200237, China; 2. Department of Mechanical \& Aerospace Engineering, University of Strathclyde, Glasgow G11XJ, UK)

\begin{abstract}
Lithium-ion batteries are widely used as energy storage and dynamic power, while the capacity life of battery is one of the key factors affecting its further application. The electrochemical-mechanical multi-field coupling effect of the lithium-ion batteries during the cyclic charging and discharging process cause the damage accumulation for the electrode materials, thereby deteriorates the mechanical stability of the electrode materials, leading to multi-scale damage to the electrode materials, ultimately declining the battery life. In this study, the multi-scale failure behavior of $\mathrm{LiNi}_{x} \mathrm{Co}_{y} \mathrm{Mn}_{z} \mathrm{O}_{2}(\mathrm{NCM})$ cathode materials were summarized through our previous research, and the experimental and simulation analysis method for studying the damage of electrode material are introduced systematically to provide reference for selecting damage analysis methods at different scales. In addition, the failure mechanisms of NCM cathode materials at the scale of active particles and electrode coating were studied in-depth based on combination of experimental and simulated analysis, including electrochemical experimental of lithium-ion
\end{abstract}

收稿日期：2021-12-20；收到修改稿日期：2022-02-08; 网络出版日期：2022-02-16

基金项目：中央高校基本科研业务费专项资金(JKG01211523); 111 引智计划(B13020); 国家自然科学基金(52150710540) Fundamental Research Funds for the Central Universities (JKG01211523); Higher Education Discipline Innovation Project (111 Project)(B13020); National Natural Science Foundation of China (52150710540)

作者简介：陈 荣(1996-), 女，博士. E-mail: yingchen96@ecust.edu.cn CHEN Ying (1996-), female, PhD. E-mail: yingchen96@ecust.edu.cn

通信作者: 奕伟玲，教授. E-mail: luan@ecust.edu.cn; 陈浩峰，教授.E-mail: haofeng.chen@ecust.edu.cn LUAN Weiling, professor. E-mail: luan@ecust.edu.cn; CHEN Haofeng, professor. E-mail: haofeng.chen@ecust.edu.cn 
batteries, extended finite element method (XFEM), linear matching method (LMM) framework. The research work provides important guidance for the mechanism analysis of multi-scale failure behavior and microstructure modification of electrode materials.

Key words: lithium-ion battery; cathode; stress field; multi-scale failure; life degradation

随着应用领域日益广泛, 锂离子电池的循环寿 命引起越来越多研究者的关注。电池寿命衰退既是 电化学过程, 也涉及到力学关键问题 ${ }^{[1-2]}$ 。充放电过 程是 $\mathrm{Li}^{+}$在正负极之间不断循环嵌入-脱出的过程, 在锂化-脱锂过程中伴随着摩尔体积的变化, 使得 极片中的 $\mathrm{Li}^{+}$浓度分布不均匀，导致材料发生往复 收缩与膨胀, 这种电化学-力学的耦合作用会形成 扩散诱导应力场，而这种应力是导致电极材料机械 失效的直接原因 ${ }^{[3-4]}$ 。此外, 在长时间电化学循环过 程中，电极内部的扩散诱导应力也会影响电池的扩 散过程, 导致电池容量的持续损失。

扩散诱导应力最早是由 Prussin ${ }^{[5]}$ 通过类比温度 梯度引起的热应力得到, 之后人们广泛研究了活性 颗粒和薄膜极片在充放电过程中扩散诱导应力的演 化过程。Huggins 和 $\mathrm{Nix}^{[6]}$ 首先建立了一维模型来分 析活性颗粒的断裂机制。Christensen 和 Newman ${ }^{[7]}$ 提出了一种耦合扩散一应力模型用于计算球形活性 颗粒的应力。在此基础上, Zhao 等 $^{[8]}$ 运用扩散动力 学和断裂力学相结合的方法, 研究了锂离子电池电 极断裂的影响因素。当前, 对扩散诱导应力作用下 电极材料损伤破坏模拟主要基于断裂力学理论或内 聚力模型进行强度失效分析, 且集中在其弹性应力 和变形上，而对不同尺度的电极材料循环塑性行为 缺乏关注。

当电极经受连续的循环充放电载荷时, 结构会 发生诸如安定、棘轮和低周疲劳等循环塑性行为。 安定行为可以进一步分为弹性安定行为和塑性安定 行为(交变塑性)。棘轮是由于塑性变形增量的累积 导致的电极材料结构塑性破坏，而疲劳会导致局部 裂纹的萌生和扩展。材料在循环载荷下的塑性屈服 是发生棘轮和低周疲劳的必要条件, 并且与充放电 过程材料的塑性流动有关 ${ }^{[9-10]}$ 。

本文系统概括了团队前期在电极材料失效方面 的研究工作和研究过程中的多尺度评估方法, 旨在 进一步分析循环充放电过程中正极材料在颗粒、不 同极片尺度下的损伤机理及循环塑性失效行为，以 及如何不同在尺度下选取对应的实验及有限元分析 方法, 结合电化学循环实验表征、扩展有限元分析 法(XFEM)、线性匹配法(LMM)等, 为进一步指导电 极材料的失效分析及结构改性提供理论参考。

\section{1 多尺度失效行为}

为探究正极材料的失效行为, 对 NCM523/Li 扣 式半电池循环至失效后拆解进行正极材料的离位 SEM 表征。研究发现正极材料 NCM523 多次电化 学循环后, 在电化学-力学耦合作用下引起的扩散 诱导应力使得 NCM523 发生了多尺度损伤, 出现正 极活性材料内部裂纹、活性颗粒破裂或与粘接剂脱 粘、活性层与集流体分层等现象(图 1)。

扩散诱导应力引起的正极材料跨尺度的力学损 伤，一方面直接破坏了多种电子和离子通路，如活 性物质颗粒内部产生裂纹影响内部电子和离子通路; 活性颗粒破裂或与粘接剂脱粘导致游离活性材料丧 失电子通路; 活性层与集流体分层使电子通路发生 改变导致电池阻抗增加。另一方面扩散诱导应力导 致正极材料开裂，使活性物质产生的新表面与电解 液接触，在一定电压下电解液分解消耗更多的锂离 子，使电池容量下降。这些由扩散诱导应力引起的 电化学-力学耦合驱动的电极材料多尺度力学损伤, 严重破坏电极材料的结构完整性，极大缩短了电极 的使用寿命，最终导致电池失效 ${ }^{[11-12]}$ 。
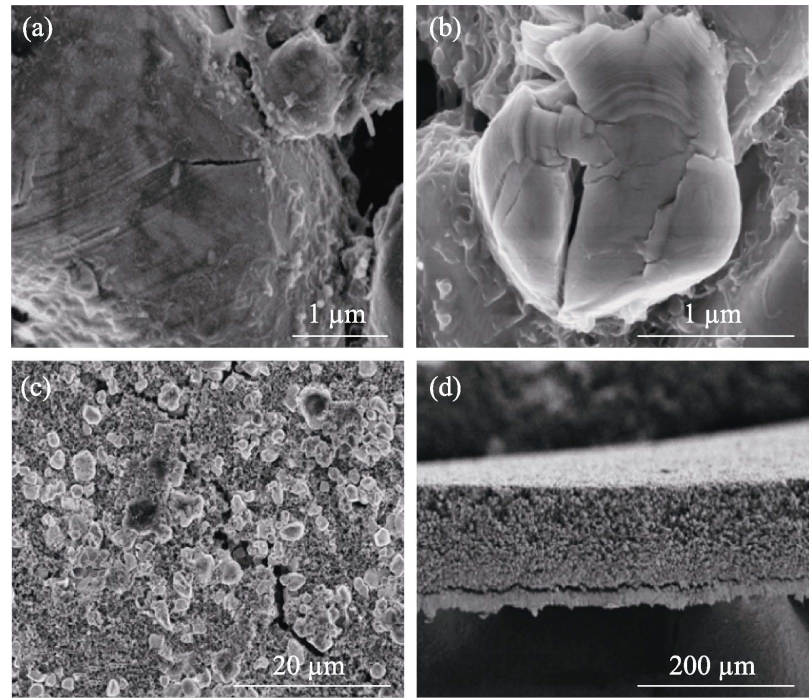

图 1 NCM523 循环后发生多尺度损伤

Fig. 1 Multi-scale damage of NCM523 after electrochemical cycles

(a) In-particle cracks; (b) Particle breakage; (c) Large cracks in electrode material; (d) Delamination between active material and current 


\section{2 数值模拟方法}

\section{1 扩散诱导应力计算}

对锂离子在循环充放电过程中的扩散诱导应力 类比于热应力进行分析 ${ }^{[5]}$ 。为了便于计算扩散一应力 耦合, 求解等效热方程并进行耦合热应力分析, 通 过开发一组 USDFLD-UMATHT 子程序在 ABAQUS ${ }^{[13]}$ 中实现扩散应力耦合分析。

对于热流, 热方程遵循热传导和能量守恒的物 理定律:

$$
k \frac{\partial u}{\partial t}+\nabla q=\phi
$$

其中, $k$ 是结合了热扩散系数、密度和比热 $\left(k=\alpha \rho C_{\mathrm{s}}\right)$ 的导热系数, $u$ 表示温度, $t$ 是时间, $q$ 是热 通量, 描述在单位时间单位面积上的能量流动, $\phi$ 定 义为内部热源。

考虑到扩散过程, 它遵循质量守恒定律:

$$
\frac{\partial c}{\partial t}+\nabla J=0
$$

式中, $c$ 为锂离子扩散浓度, $t$ 为时间, $J$ 为扩散通 量, 即单位时间单位面积内流过的物质的量。假设 $u=c, \phi=0, q=J, k=1$, 质量扩散行为可以类比为热传 导过程。

基于经典菲克第一定律, 在不考虑应力影响情 况下, $J$ 通量的表达式如下:

$$
J=-D_{\mathrm{f}} \nabla c
$$

其中, $D_{\mathrm{f}}$ 为扩散系数, 可以表达如下:

$$
D_{\mathrm{f}}=D_{0} \exp \left(\frac{\Omega \sigma_{\mathrm{h}}}{R T}\right)
$$

其中, $D_{0}$ 是无应力状态下的扩散系数, $D_{\mathrm{f}}$ 为扩散 系数, $\Omega$ 为锂离子的偏摩尔体积, $R$ 是气体常数, $T$ 是绝对温度, $\sigma_{\mathrm{h}}$ 是与扩散诱导应力相关的静水 应力, 可以由积分点上主应力分量的平均值计算得 到: $\sigma_{\mathrm{h}}=\left(\frac{\sigma_{1}+\sigma_{2}+\sigma_{3}}{3}\right)$ 。

与扩散过程相关的扩散系数 $D_{\mathrm{f}}$ 嵌入应力效应 中, 其边界条件如下:

$$
J=-D_{\mathrm{f}}\left(\nabla c-\frac{\Omega c}{R T} \nabla \sigma_{\mathrm{h}}\right)=\frac{i_{\mathrm{n}}}{F}
$$

其中, $i_{\mathrm{n}}$ 为电极表面的电流密度 (假设电流密度为常 数), $c$ 表示锂离子扩散浓度, $F$ 为法拉第常数。

\section{2 扩展有限元法}

基于耦合扩散一应力分析, 可采用 ABAQUS $\mathrm{XFEM}^{[14]}$ 和粘结单元来模拟活性颗粒的裂纹萌生扩
展及断裂过程。本文选用 XFEM 进行分析, 裂纹尖 端附近渐近函数用于描述裂纹尖端区域的应力奇异 性, 引入间断函数来表示裂纹面处位移跳跃。并且 XFEM 能模拟材料中裂纹沿任意路径萌生和扩展, 无需对裂纹尖端重新进行网格划分。这些优点使得 XFEM 成为分析颗粒裂纹萌生及扩展的有效方法。

\section{3 线性匹配法}

扩散诱导应力可与 $\mathrm{LMM}$ 结合用于评估 $\mathrm{NCM}$ 正极材料在不同尺度下的安定、棘轮边界和低周疲 劳寿命。LMM 是一种模拟实际非线性材料弹塑性 力学响应的先进直接分析的方法, 主要包括模量 随空间和时间变化的大量迭代线性分析, 相关安 定棘轮极限的详细计算过程可以参考团队前期工 作 ${ }^{[15-16]}$ 。

基于 ABAQUS 以及 $\mathrm{LMM}$ 插件, 可系统研究 $\mathrm{NCM}$ 正极材料在不同尺度下基于应力场的棘轮边 界和循环破坏行为。

\section{3 三元正极材料失效行为分析}

在对正极材料进行失效分析时，考虑其不同尺 度下失效机理差异, 可从活性颗粒尺度以及极片尺 度进行实验研究及有限元模拟分析。

\section{1 电极颗粒断裂评估}

采用 ABAQUS XFEM 对 NCM 活性颗粒进行裂 纹萌生及扩展分析, 并假设其为球形(图 2)。在进行 颗粒的损伤断裂分析时, 考虑到电极颗粒被粘结材 料包围且粘结材料的刚度远小于电极颗粒, 力学边 界条件设置为颗粒的无牵引表面。采用双线性牵引一 分离损伤模型和最大主应力作为损伤判据, 计算过 程中裂纹会根据应力场的演变进行扩展, 其力学断 裂机理为: 将最大主应力作为裂纹萌生的阈值, 当 最大主应力超过材料抗拉强度时会发生损伤。

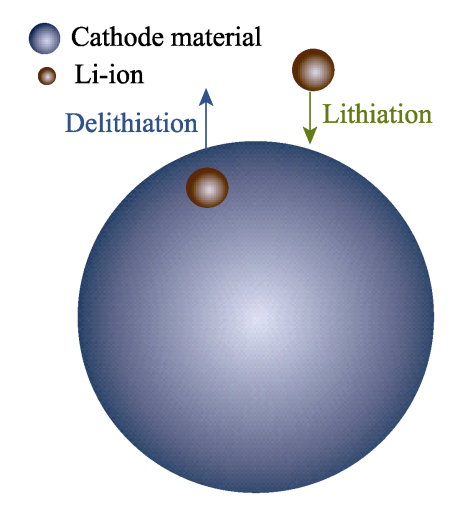

图 2 活性颗粒脱嵌锂示意图

Fig. 2 Schematic illustration of active particle during the lithiation/ delithiation process 


\subsection{1 颗粒裂纹萌生扩展过程}

在理想状态下，对球对称的几何模型施加各向 同性的材料应力, 会产生球对称的应力场, 将最大 主应力破坏准则与最大主应力分布相关联, 裂纹损 伤将从颗粒中心开始(图 3)。当分析颗粒裂纹萌生时, 可采用在颗粒表面任意点施加远小于扩散诱导应力 的集中力, 作为微小应力扰动来实现非均匀应力场, 本团队施加的微小应力扰动大小为扩散诱导应力的 $1 / 100^{[17]}$ 。

在计算应力扰动的扩散诱导应力场后, 可进一 步分析活性颗粒裂纹的萌生扩展过程。图 3 展示了 直径 $3 \mu \mathrm{m}$ 颗粒在 $0.37 \mathrm{~A} / \mathrm{m}^{2}$ 的电流密度下嵌锂和脱 锂阶段损伤情况。在嵌锂阶段(图 3(a)), 裂纹在颗粒 中心处萌生并逐渐发展，370 $\mathrm{s}$ 时迅速向外对称扩 展。之后, 裂纹缓慢发展, 直至嵌锂阶段结束, 且未 穿透颗粒。在脱锂阶段(图 3(b)), $204 \mathrm{~s}$ 时颗粒表面出 现了新裂纹，同时内部裂纹保持稳定。随着内部裂 纹扩展, 新形成的裂纹在颗粒表面迅速扩展, 最终 裂纹交汇导致颗粒的贯穿性断裂。

\subsection{2 颗粒断裂边界}

当单元内最大主应力超过临界破坏应力时, 裂 纹会逐渐扩展直至达到临界断裂能, 相应粘结单元

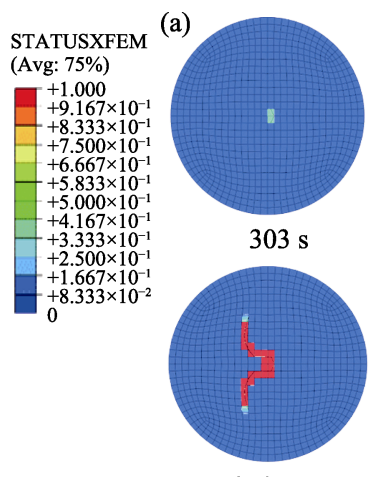

$370.7 \mathrm{~s}$

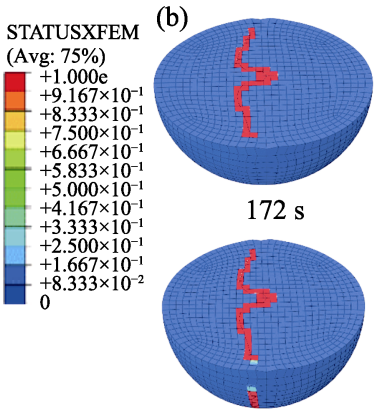

$204.9 \mathrm{~s}$

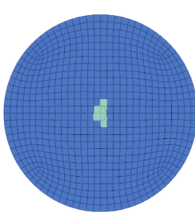

$370.4 \mathrm{~s}$

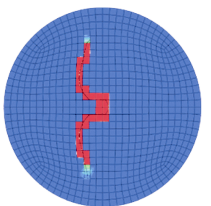

$370.8 \mathrm{~s}$

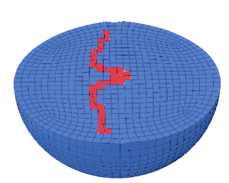

$204.7 \mathrm{~s}$

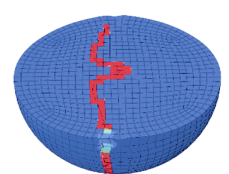

$204.9 \mathrm{~s}$

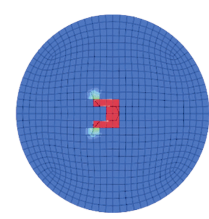

$370.6 \mathrm{~s}$

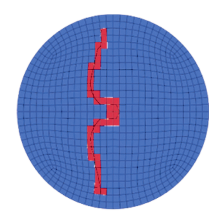

$6157 \mathrm{~s}$

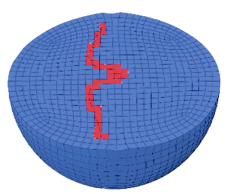

$204.9 \mathrm{~s}$

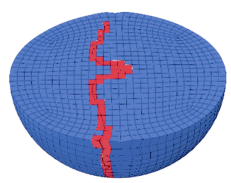

$215 \mathrm{~s}$
图 3 直径为 $3 \mu \mathrm{m}$ 颗粒在电流密度 $0.37 \mathrm{~A} / \mathrm{m}^{2}$ 下裂纹萌生扩 展过程 ${ }^{[17]}$

Fig. 3 Crack initiation and propagation of active particle of diameter of $3 \mu \mathrm{m}$ under the current density of $0.37 \mathrm{~A} / \mathrm{m}^{2[17]}$

(a) Lithiation process; (b) Delithiation process. The variable STATUSXFEM presents the status of element, and it varies 0 (non-damage status) or 1 (completely cracked)
完全断裂。随着脱锂-嵌锂的进行, 更多粘结单元出 现断裂现象, 这会导致颗粒整体发生力学破坏。

对不同尺寸的 NCM 颗粒的裂纹萌生规律进一 步分析，可以绘制出完整的裂纹萌生边界(黑色实 线)和颗粒断裂边界(黑色虚线), 如图 4 所示。整个 裂纹准则图分为三个区域：断裂区域、裂纹扩展区 域和稳定区域。其中黑色实线为不同尺寸颗粒裂纹 萌生的临界电流密度，可以发现活性颗粒的临界电 流密度随着颗粒尺寸的增加而减小。通过电化学循 环后离位 SEM 表征结果(图 5), 可以发现大颗粒上 有多条明显裂纹，而小颗粒上未发现明显裂纹，这 一结论与上述模拟结果一致。这也说明减小颗粒的 平均尺寸有利于提高 NCM 颗粒的力学稳定性。

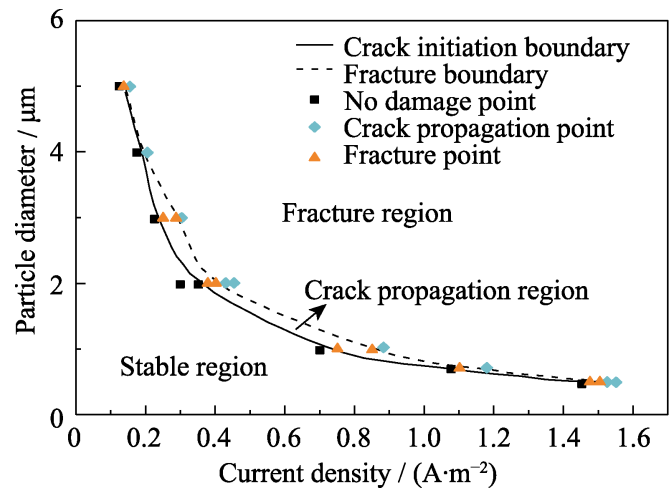

图 4 NCM 颗粒断裂准则图 ${ }^{[17]}$

Fig. 4 Integrated fracture criteria diagram for NCM particles ${ }^{[17]}$
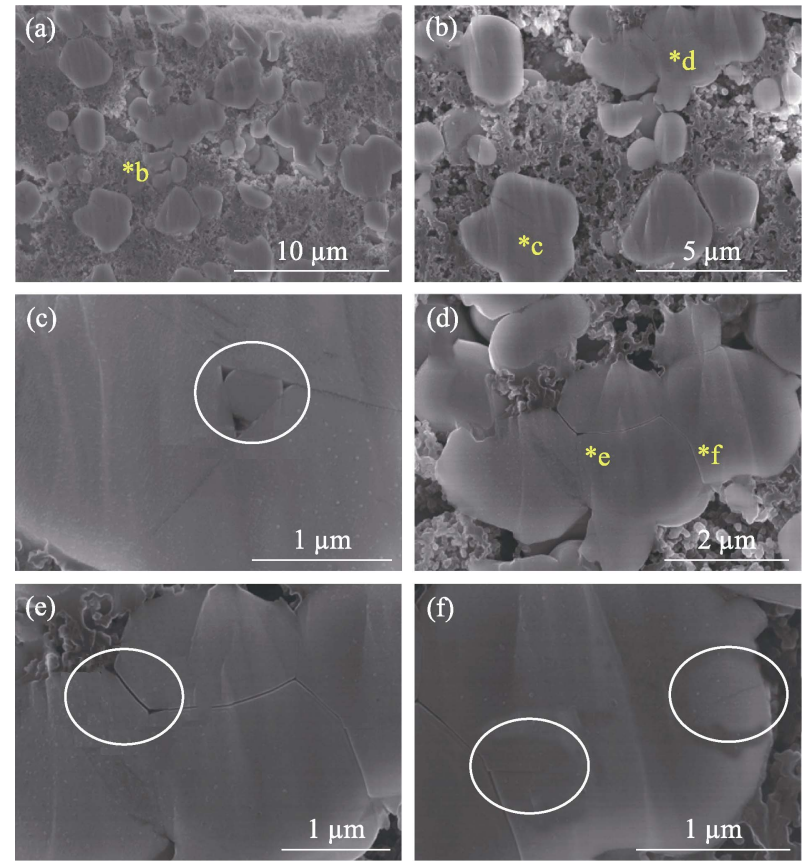

图 5 电化学循环后 NCM 横截面的 SEM 照片

Fig. 5 SEM images of NCM cathode after cyclic chargingdischarging test

Different magnification SEM images showing the cracks in the big or small paticles 
基于以上分析结果, 可以获得颗粒在裂纹萌生 状态下的直径 $d_{\mathrm{i}}$ 及颗粒断裂状态下的直径 $d_{\mathrm{f}}$, 与 相应临界电流密度 $i_{\mathrm{n}}$ 之间的拟合关系式:

裂纹萌生： $d_{\mathrm{i}}=0.7424 i_{\mathrm{n}}^{-0.978}$

颗粒断裂: $d_{\mathrm{f}}=0.8155 i_{\mathrm{n}}^{-0.979}$

\section{2 极片循环塑性行为分析}

为研究 NCM 极片在电化学循环过程中的力 学损伤失效行为, 需先建立有限元分析模型(图 6) 来计算扩散诱导应力场, 采用的是理想弹塑性模 型 ${ }^{[7,18-19]}$ 。为了简化模型，该几何模型中没有包含 集流体，但考虑到集流体对活性层底部的约束以及 集流体的杨氏模量远远大于活性层的杨氏模量, 模 型中对 NCM 活性层底部完全约束, 会使结果偏保 守但保持足够的计算精度 ${ }^{[20]}$ 。

\subsubsection{NCM 活性层的安定棘轮边界}

基于扩散诱导应力的计算方法得到 NCM 活性 层在充放电过程中的扩散诱导应力场, 再通过 ABAQUS LMM 插件分析, 可以得到 NCM 活性层 的安定棘轮边界。图 7 为考虑了厚度影响的 $\mathrm{NCM}$ 极片安定极限和棘轮极限边界，其中 $\Delta \theta / \Delta \theta_{0}$ 、 $p / p_{0}$ 分别是归一化的循环充放电倍率和压力, $\Delta \theta_{0}$ 和 $p_{0}$ 的设定均与充放电实验负载条件一致。值 得注意的是，反向塑性极限与棘轮极限都随着活性 层厚度增加而增大, 这说明在循环扩散诱导应力下 较薄的活性层更危险。

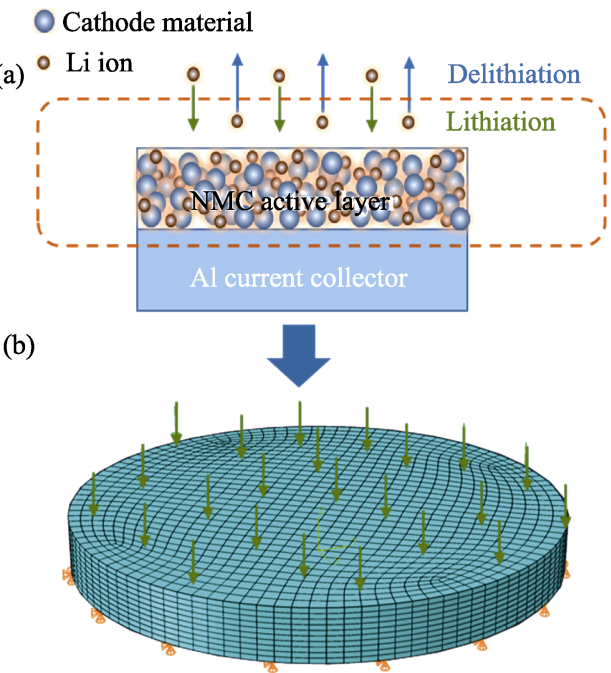

图 6 (a)电极在嵌锂/脱锂过程的示意图(NCM 活性层涂覆在 $\mathrm{Al}$ 集流体上)，(b)边界条件和在锂化阶段对电极施加的负载 ${ }^{[20]}$ Fig. 6 (a) Schematic illustration of electrode during the lithiation/ delithiation process, whereby NCM active layer being coated on the $\mathrm{Al}$ current collector, and (b) boundary condition and the load applied to electrode during the lithiation stage ${ }^{[20]}$

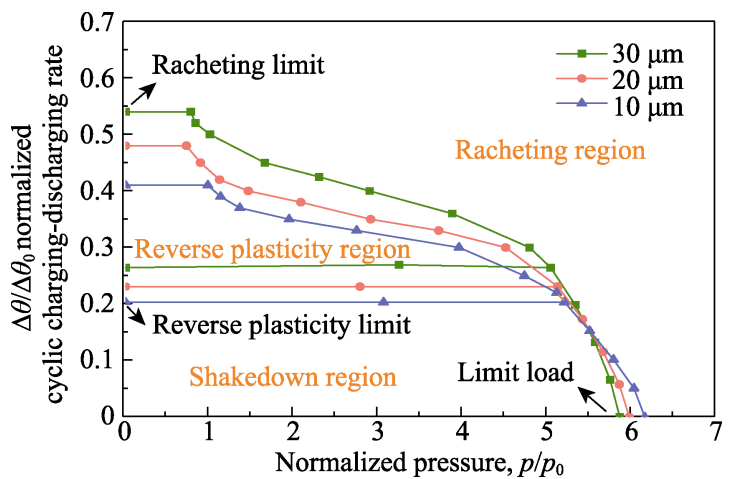

图 $7 \mathrm{NCM}$ 厚度为 $10 、 20 、 30 \mu \mathrm{m}$ 的安定棘轮边界 ${ }^{[20]}$

Fig. 7 Shakedown and ratcheting limit curves of NCM material with varying thickness of 10,20 and $30 \mu \mathrm{m}^{[20]}$

\subsubsection{NCM 活性层的低周疲劳寿命}

基于 Manson-Coffin 关系 ${ }^{[21]}$, 塑性应变与疲劳 寿命之间的关系可以表示为:

$$
\lg \frac{\Delta \varepsilon_{\mathrm{p}}}{2}=k \lg \left(2 N_{\mathrm{f}}\right)+\lg \varepsilon_{\mathrm{f}}^{\prime}
$$

其中, $\frac{\Delta \varepsilon_{\mathrm{p}}}{2}$ 是塑性应变幅值, $2 N_{\mathrm{f}}$ 是疲劳寿命, $\varepsilon_{\mathrm{f}}^{\prime}$ 为疲劳延性指数, $k$ 为疲劳延性系数。

为了更简单明了地描述电极材料的低周疲劳寿 命，可用如下公式进行拟合:

$$
\lg \Delta \varepsilon_{\mathrm{p}}=a \lg N_{\mathrm{f}}+b
$$

其中, $a 、 b$ 为疲劳常数。变量 $\Delta \varepsilon_{\mathrm{p}}$ 可以基于有限元 计算分析得到。 $N_{\mathrm{f}}$ 为电极材料的低周疲劳寿命, 可 由电化学循环测试确定。该参数化的应变寿命曲线 可用于预测 NCM 在其他充放电倍率下的寿命, 并 能与实验循环容量衰减对比。

\subsubsection{NCM 活性层在充放电下的循环塑性行为}

通过数值分析结果和实验测试结果的相互对照, 可以进一步研究在实际电化学测试条件下的 NCM 失效机理。例如, 厚度为 $20 \mu \mathrm{m}$ 的 $\mathrm{NCM}$ 活性层在 $1 C$ 充放电倍率和 $19.15 \mathrm{MPa}$ 恒定压力下的循环塑性 行为(对应于图 7 中的载荷点 $(1,1))$ 。显然，载荷工 况 $(1,1)$ 位于棘轮区域, 在该工况下既存在疲劳又具 有棘轮损伤。图 8（a）为实际测试工况下 $\mathrm{NCM}$ 材 料的塑性应变范围和棘轮应变云图。其中, 棘轮应 变定义为稳态周期内循环塑性应变的净累积。积分 点的塑性应变范围最大值为 0.146 , 位于中心; 而棘 轮应变最大值为 $1.68 \times 10^{-2}$, 位于边缘。这表明正极 的上表面中心表现出疲劳破坏, 而由于塑性应变积 累, 上表面的边缘表现出棘轮行为。因此, 裂纹萌生 和扩展主要发生在正极活性层中心, 而边缘塑性变 形累积会引发棘轮现象, 导致活性层边缘形成渐增 


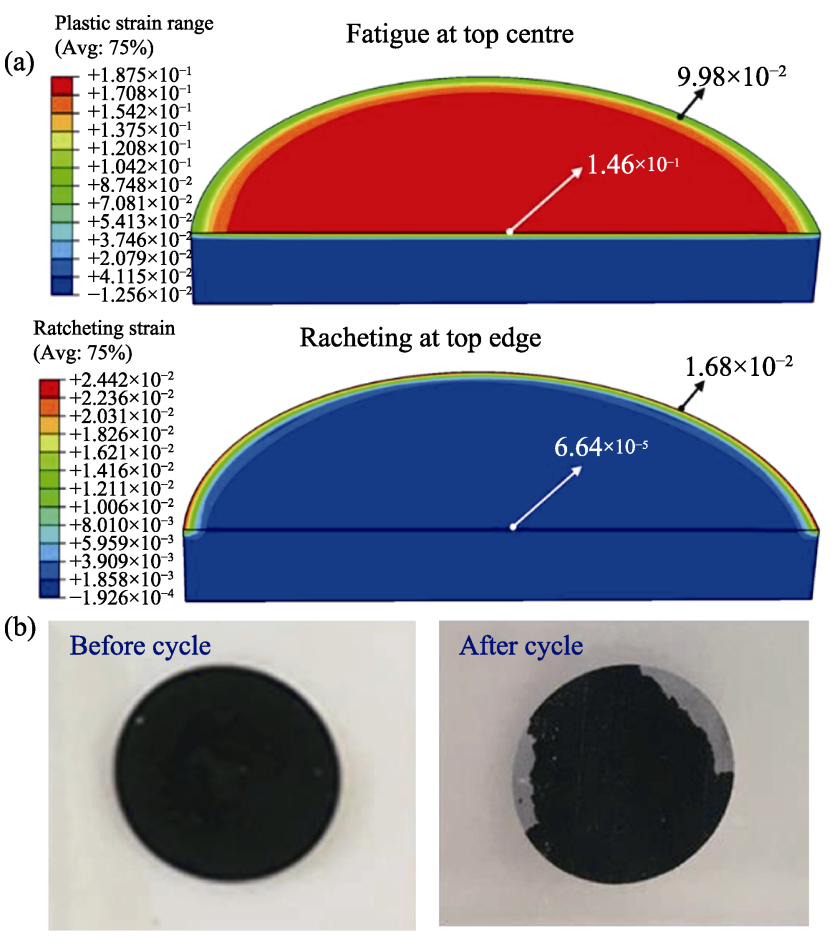

图 8 (a)厚度为 $20 \mu \mathrm{m}$ 的 $\mathrm{NCM}$ 活性层在载荷点 $(1,1)$ 下的塑 性应变范围和棘轮应变云图, (b)充放电循环实验前(左)和实 验后(右)NCM 正极照片 ${ }^{[20]}$

Fig. 8 (a) Contour map of plastic strain range and ratcheting strain of NCM active layer of $20 \mu \mathrm{m}$ thickness for load point $(1,1)$ and (b) picture of NCM cathode before (left) and after (right) cyclic charging-discharging test ${ }^{[20]}$

\section{性塑性垮塌。}

循环充放电实验后拆解，也观察到棘轮机制 (图 8(b)), 即活性层与集流体的剥离倾向于发生在 正极表面的边缘，这与数值分析结果一致。

\section{4 结论}

基于 ABAQUS 数值模拟, 结合电化学循环实 验结果, 开展了锂离子电池 $\mathrm{NCM}$ 材料在不同尺度 下的循环失效行为研究。针对不同失效模式采用不 同的研究方法，获得如下结论：

1) NCM 活性颗粒裂纹从中心处萌生扩展, 当 达到临界断裂能, 发生颗粒断裂。颗粒的断裂过程 与其尺寸以及所施加电流密度有关。当大多数颗粒 处于断裂区域时，将会发生跨尺度的失效行为(极 片尺度大裂纹或活性层与集流体脱粘)。其中, NCM 极片中心处存在疲劳现象易产生裂纹, 边缘处存在 棘轮机制导致整体脱粘。

2)基于扩散应力耦合分析及 ABAQUS XFEM 可进行活性颗粒裂纹萌生扩展评估，从而建立临界 裂纹萌生扩展边界。基于扩散诱导应力场以及 LMM
分析可以得到电极材料不同尺度下的安定棘轮边界, 预测疲劳寿命并分析循环塑性失效机理。

\section{参考文献:}

[1] HENDRICKS C, WILLIARD N, MATHEW S, et al. A failure modes, mechanisms, and effects analysis (FMMEA) of lithium-ion batteries. Journal of Power Sources, 2015, 297: 113-120.

[2] XU R, YANG Y, YIN F, et al. Heterogeneous damage in Li-ion batteries: experimental analysis and theoretical modeling. Journal of the Mechanics and Physics of Solids, 2019, 129: 160-183.

[3] WANG Y N, LI H, WANG Z K, et al. Progress on failure mechanism of lithium ion battery caused by diffusion induced stress. Journal of Inorganic Materials, 2020, 35(10): 1071-1087.

[4] RYU H H, NAMKOONG B, KIM, J H, et al. Capacity fading mechanisms in Ni-rich single-crystal NCM cathodes. ACS Energy Letters, 2021, 6(8): 2726-2734.

[5] PRUSSIN S. Generation and distribution of dislocations by solute diffusion. Journal of Applied Physics, 1961, 32(10): 1876-1881.

[6] HUGGINS R A, NIX W D. Decrepitation model for capacity loss during cycling of alloys in rechargeable electrochemical systems. Ionics, 2000, 6(1): 57-63.

[7] CHRISTENSEN J, NEWMAN J. Stress generation and fracture in lithium insertion materials. Journal of Solid State Electrochemistry, 2006, 10(5): 293-319.

[8] ZHAO K, PHARR M, VLASSAK J J, et al. Fracture of electrodes in lithium-ion batteries caused by fast charging. Journal of Applied Physics, 2010, 108(7): 473

[9] TAKAMURA T, OHARA S, UEHARA M, et al. A vacuum deposited Si film having a Li extraction capacity over $2000 \mathrm{mAh} / \mathrm{g}$ with a long cycle life. Journal of Power Sources, 2004, 129(1): 96-100.

[10] ZHAO K, TRITSARIS G A, PHARR M, et al. Reactive flow in silicon electrodes assisted by the insertion of lithium. Nano Letters, 2012, 12(8): 4397-4403.

[11] LEE Y K, SONG J, PARK J, et al. Multi-scale coupled mechanicalelectrochemical modeling for study on stress generation and its impact on multi-layered electrodes in lithium-ion batteries. Electrochimica Acta, 2021, 389: 138682.

[12] FERRARO M E, TREMBACKI B L, BRUNINI V E, et al. Electrode mesoscale as a collection of particles: coupled electrochemical and mechanical analysis of NMC cathodes. Journal of the Electrochemical Society, 2020, 167(1): 013543.

[13] ABAQUS. ABAQUS/CAE user's guide, Version 6.14. Dassault Syst` emes Simulia Corp., Providence, RI, USA, 2014.

[14] BELYTSCHKO T, BLACK T. Elastic crack growth in finite elements with minimal remeshing. International Journal for $\mathrm{Nu}$ merical Methods in Biomedical Engineering, 1999, 45(5): 601-620.

[15] CHEN H, PONTER A. Shakedown and limit analyses for 3-D structures using the linear matching method. International Journal of Pressure Vessels and Piping, 2001, 78: 443-451.

[16] CHEN H, PONTER A. A direct method on the evaluation of ratchet limit. Journal of Pressure Vessel Technology, 2010, 132(4): 041202.

[17] ZHU X, CHEN Y, CHEN H, et al. The diffusion induced stress and cracking behavior of primary particle for Li-ion battery electrode. International Journal of Mechanical Sciences, 2020, 178: 105608.

[18] ZHANG J, LU B, SONG Y, et al. Diffusion induced stress in layered Li-ion battery electrode plates. Journal of Power Sources, 2012, 209: 220-227.

[19] NADIMPALLI S P V, SETHURAMAN V A, ABRAHAM D P, et al. Stress evolution in lithium-ion composite electrodes during electrochemical cycling and resulting internal pressures on the cell casing. Journal of The Electrochemical Society, 2015, 162: A2656-A2663.

[20] CHEN Y, LUAN W, CHEN H. Shakedown, ratcheting and fatigue analysis of cathode coating in lithium-ion battery under steady charging-discharging process. Journal of the Mechanics and Physics of Solids, 2021, 150: 104366.

[21] MANSON S S. Fatigue: a complex subject-some simple approximations. Experimantal Mechanics, 1965, 5: 193-226. 


\section{我与郭景坤先生}

1995 1998 年, 我师从郭先生攻读博士学位, 研究纳米化介电材料的合成及性能。这个选题在当时非常超前 和创新, 体现了先生在学术领域的高瞻远瞩, 也让我此生与纳米功能材料结下了不解之缘。毕业后我留在高性

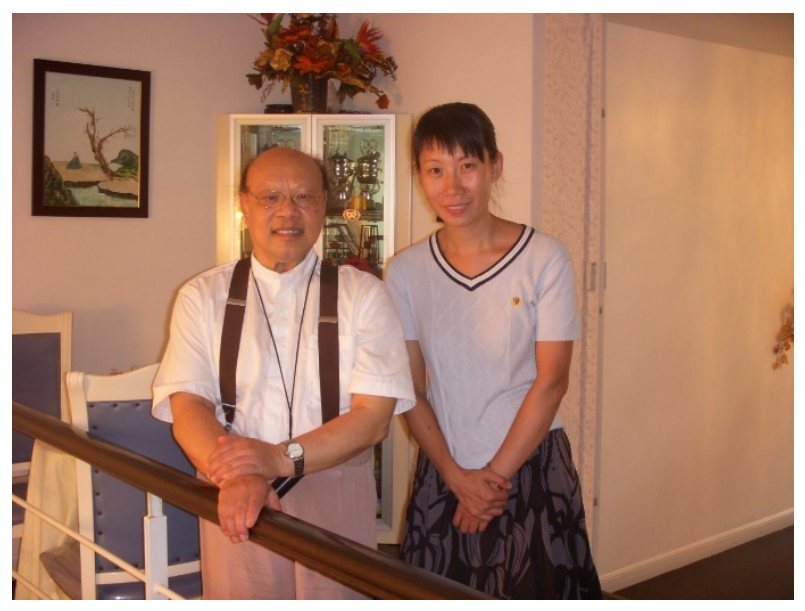

左: 郭景坤先生; 右: 奕伟玲 能陶瓷和超微结构国家重点实验室工作了两年。那时，郭 先生担任着中国科学院上海硅酸盐研究所所长, 公务特别 繁忙, 但他依然非常关心学生的学习、工作和生活, 经常 召集我们到他的办公室汇报工作, 了解我们的学习状态, 逢年过节请我们去家里聚餐。同时还让我参与国际会议的 会务工作，协助接待外宾并担任会议翻译工作，这使我开 阔了眼界，见识了诸多学术大咖的风采，并锻炼了国际交 往的能力, 让我获益终生。

如今, 我担任研究生导师已经二十年, 在学术研究和 学生培养方面也取得了小小的成绩。每每忆及和先生在一 起的点点滴滴, 满怀感恩。先生的教诲, 至今受益, 终生 难忘! 